\title{
Evaluation of the Dynamic Accuracy of a GPS Receiver*
}

\author{
—Is Dynamic Accuracy the Same as Static Accuracy?-
}

Tadashi CHOSA ${ }^{* 1}$, Masaaki OMINE ${ }^{* 2}$, Kenji ITANI $^{* 3}$, Reza EHSANI $^{* 4}$

\begin{abstract}
An experimental testbed and a data analysis procedure were developed to evaluate the dynamic performance of global positioning system (GPS) receivers. Receiver I - radio beacons differential correction based GPS receiver and Receiver II - satellite differential correction based GPS receiver were evaluated. Under dynamic conditions, the position errors were 1.39 and $1.71 \mathrm{~m}$, the speed errors were 0.067 and $0.068 \mathrm{~m} / \mathrm{s}$, and the direction errors were $9.7^{\circ}$ and $16.9^{\circ}$ for the receivers I and II, respectively. The results indicated that the trends in the dynamic accuracy of GPS receivers were completely different from those for static accuracy. The study signifies the need for evaluating the GPS positioning errors under static as well as dynamic conditions to correct the errors.

[Keywords] dynamic accuracy, error, evaluation, fixture test, global positioning system, static accuracy
\end{abstract}

\section{Introduction}

The global positioning system (GPS) is widely used in many practical applications as well as in agricultural research, where it has become an essential technology. Yield mapping, guidance systems, and other technologies using GPS have already reached the commercial market. Autonomous vehicles (Nagasaka et al., 2004) and more accurate variable-rate technology (VRT) (Chosa et al., 2007a) require a very accurate GPS. Knowledge of the accuracy of GPS receivers is important in developing and assessing such precision agriculture vehicles. The Institute of Navigation (1997) has described static accuracy standards for GPS receivers. However, most agricultural applications using GPS receivers involve dynamic conditions. Therefore, the dynamic performance of GPS receivers must be evaluated in addition to their static performance.

Several dynamic tests on GPS receivers for agricultural applications have been performed.

Ehsani et al. (2003) tested low-cost GPS receivers for dynamic accuracy and the dynamic accuracy of five receivers was compared. The cross-track error ranged from 0.5 to $2 \mathrm{~m}$. This result confirmed the existence of direction bias, and Han et al. (2004) analyzed the pass-to-pass accuracy of GPS receivers in vehicle tests using eight differential GPS (D-GPS) receivers. The pass-to-pass accuracies of these receivers were quite different from each other, ranging from $0.05 \mathrm{~m}$ to $0.85 \mathrm{~m}$. Taylor et al. (2004) tested dual-frequency and non-differential GPS receivers and demonstrated the periodic content of errors. The cross-track accuracy of the dual-frequency GPS was approximately $0.17 \mathrm{~m}$ and approximately $1.35 \mathrm{~m}$ for one of the non-differential GPS unit. Stombaugh et al. (2005) observed overshoot errors in GPS receivers, particularly in corners or curved paths, using a moving platform on a specially designed fixed path.

Previous researchers have evaluated mainly cross-track errors, which can be defined as the distance between the measured GPS position and the actual path (i.e., error perpendicular to the direction of travel). However, the actual error consists of the cross-track error plus the track error, i.e., the error parallel to the direction of travel. Track errors are significant in applications in which recognizing a zone change in VRT or understanding the turning point in autonomous driving is important. A velocity-based control system designed to achieve accurate operation in a muddy field affected by slippage (Chosa et al., 2007a) requires accurate

\footnotetext{
* Partly presented at the 8th International Conference on Precision Agriculture, Minnesota, USA. in July 2006 and the 44th Annual Meeting of Kanto Branch of the Japanese Society of Agricultural Machinery (JSAM), Toyama, Japan. in August 2008

*1 JSAM Member, Corresponding Author, Advanced Lowland Rice Farming Team (Hokuriku Region), National Agricultural Research Center, Present address: Institute of Agriculture, Tokyo University of Agriculture and Technology, 3-5-8, Saiwai-cho, Fuchu, Tokyo, 183-8509, Japan; E-mail: chosa@cc.tuat.ac.jp

*2 JSAM Member, Upland Farming Research Team (Kyushu Region), National Agricultural Research Center for Kyushu Okinawa Resign, 6651-2 Yokoichi-machi, Miyakonojyo, Miyazaki, 885-0091, Japan.

*3 Faculty of Maritime Sciences, Kobe University, 5-1-1 Fukaeminami-machi, Higashinada-ku, Kobe, 658-0022, Japan.

*4 Department of Agricultural and Biological Engineering, Citrus Research and Education Center, University of Florida, Lake Alfred, FL 33850, USA.
} 
velocity monitoring unaffected by track error. Presently, no standard is available for evaluating velocities through GPS receivers.

The objective of this research was to evaluate the dynamic accuracy of GPS receivers using an experimental testbed developed for the purpose. The static and dynamic accuracies of two types of GPS receivers were measured using the testbed, and differences in their performance were analyzed.

\section{Materials and Methods}

\section{Platform for testing the dynamic accuracy of GPS}

\section{receivers}

The main component of the testbed was a turntable equipped with a boom driven by a three-phase squirrel-cage induction motor (Fig. 1, 2). An inverter (FVR0.75C11S-2, Fuji Electric, Tokyo, Japan) was used to control the rotational speed of the motor by varying the frequency of the power supplied to it. Ignoring the loss of transmitted power, the speed of the turntable was nominally $1 / 120$ that of the motor. However, the turntable speed was monitored using a magnet-type detector to avoid any influence of power loss or unstable motor revolution due to a change in load.

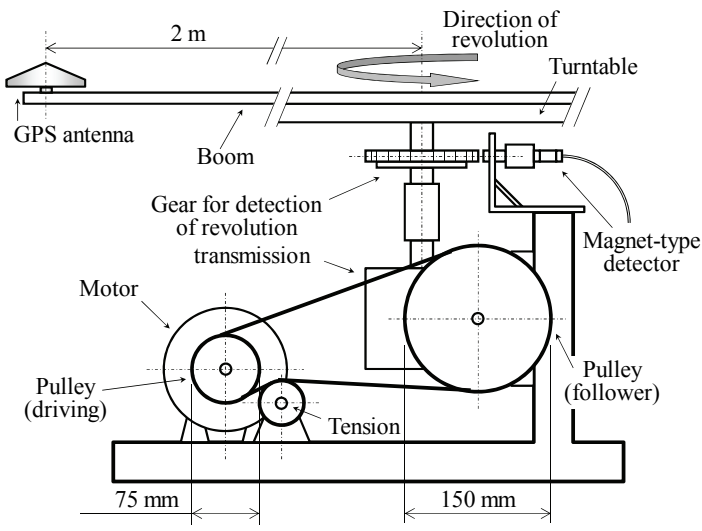

Fig. 1 Side view of the experimental testbed

A magnet-type detector (MP-981, Ono Sokki, Yokohama, Japan), containing an internal Hall element and a permanent magnet, detected the movement of the $\mathrm{S} 45 \mathrm{C}$ steel gear in the body of the turntable. The gear had 120 teeth and a module of 1. The detector obtained 120 rectangular waveforms as an analog signal during each revolution of the turntable. That is, each rectangular waveform (i.e., the peak and trough) corresponded to $1 / 120$ of a turntable revolution. The rotational speed was calculated as the average during $60 \mathrm{~s}$ of continual rotation for each condition. Although the measured rotational speed was slightly less than the theoretical value (Fig. 3), the difference confirmed that outside factors contributed to the power loss or load change did affect the speed and validated the accuracy of this detection method. The rotational speed of the turntable in this setup was proportional to the control frequency over the range from 1.0 to $48.7 \mathrm{~Hz}$ (Fig. 3). The peaks and troughs of the rectangular waveforms in these experiments had the same period when the rotational speed was constant. Each component of the rectangular waveform (i.e., each positive or negative excursion) corresponded to $1 / 240$ of a turntable revolution, and this was the resolution of the measurement.

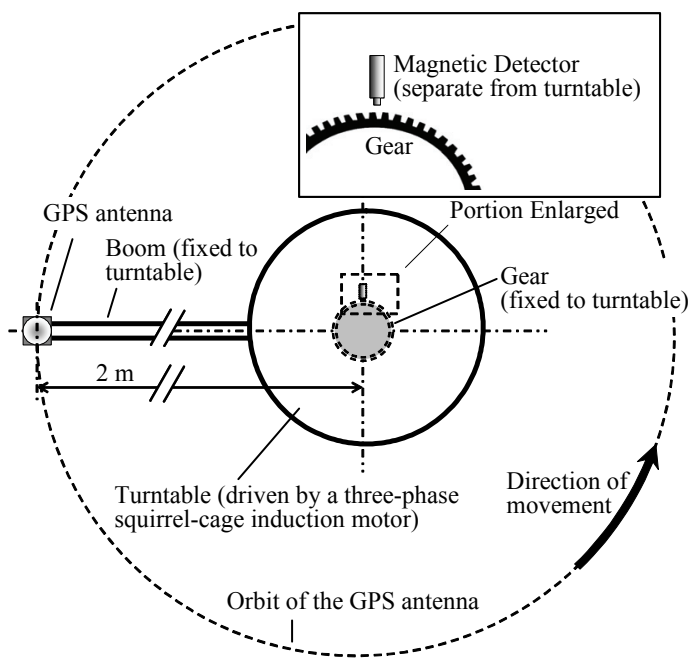

Fig. 2 Top view of the experimental testbed

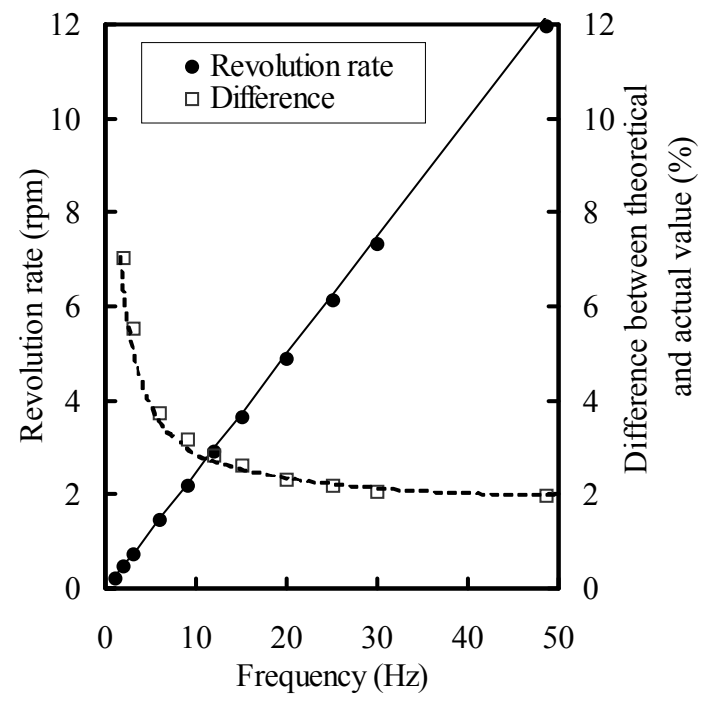

Fig. 3 Relationship between the control frequency and the rate of turntable revolution

A GPS antenna was mounted on the arm, and the receiver, computer for data storage, and battery were fixed to the turntable. A data logger collected the analog signals from the revolution detector. The rotational speed and the GPS data captured during turntable movement were compared to assess 
the dynamic accuracy of the receiver.

The peripheral velocity observed by the GPS receiver was determined from the rotational speed or angular velocity observed by the detector and the distance (radius) from the center to the antenna. For a radius of $2 \mathrm{~m}$, our setup permitted the evaluation of velocities in the range $0.05-2.50 \mathrm{~m} / \mathrm{s}$ for a control frequency of 1.0-48.7 Hz (Fig. 3). This range was sufficient for assessing the dynamic accuracy of a GPS receiver required for farm machinery.

\section{Analysis procedure}

(1) Determining the rotational speed

Figure 4 shows the data processing procedure used to determine the rotational speed. The turning of the turntable was detected by a magnet-type detector, which produced an analog rectangular waveform that was filtered and converted precisely to 0 or $5 \mathrm{~V}$. Each component of the rectangular waveform corresponds to increments of 1/240 of a turntable revolution. The relative angular position can be calculated by counting the increments. The resolution of movement depends on this step-wise increase not but the sampling interval when the rectangular waveform is larger than the sampling interval. Thus, a linear interpolation of discrete the data was processed to determine dynamic movement. Although this calculation is a simple approximation of the actual rotation, the resolution of the movement becomes much finer than $1 / 240$ of a turntable revolution. It is also possible to properly compare the GPS information following a time stamp. The angular velocity resolution depended on the resolution of the movement and the sampling interval as expressed in Eq. (1):

$$
\omega_{\text {resol }}=\theta_{\text {resol }} / \Delta t
$$

where, $\omega_{\text {resol }}$ is the resolution of the angular velocity $(\mathrm{rad} / \mathrm{s})$, $\theta_{\text {resol }}$ is the resolution of the movement (radians), and $\Delta \mathrm{t}$ is the sampling interval (s). For a monitoring period of $1 \mathrm{~s}$ and a movement resolution of $1 / 240$ of a revolution, the resolution of the angular velocity was less than $0.026 \mathrm{rad} / \mathrm{s}$ due to the interpolation process.

(2) Analysis and comparison of the GPS data

Some GPS receivers provide the speed and direction of movement directly. However, understanding how the direction is actually defined is essential; this is called the true track. The GPS direction is usually measured from north, which is geometrically upward, with a positive direction in the clockwise direction. In terms of $x y$ coordinates, the base axis is the $x$-axis, positive is to the right. We determined the direction of movement using Eq. (2).

The speed is defined by the radius of the movement of the GPS antenna and the angular velocity of the platform. The direction of the movement is geometrically calculated by the angular position in Eq. (3).

$$
\begin{aligned}
& \varphi_{1}=-\psi+(\pi / 2) \\
& \varphi_{2}=\theta+(\pi / 2),
\end{aligned}
$$

where, $\varphi_{1}$ and $\varphi_{2}$ are the direction of movement of the GPS antenna calculated by the GPS and the testbed platform, respectively; $\psi$ is the direction obtained by the GPS; and $\theta$ is the angular position of the GPS antenna obtained from the testbed platform. The turntable of the testbed moved counterclockwise (Fig. 1, 2). The direction is the positive direction in Eqs (2) and (3).

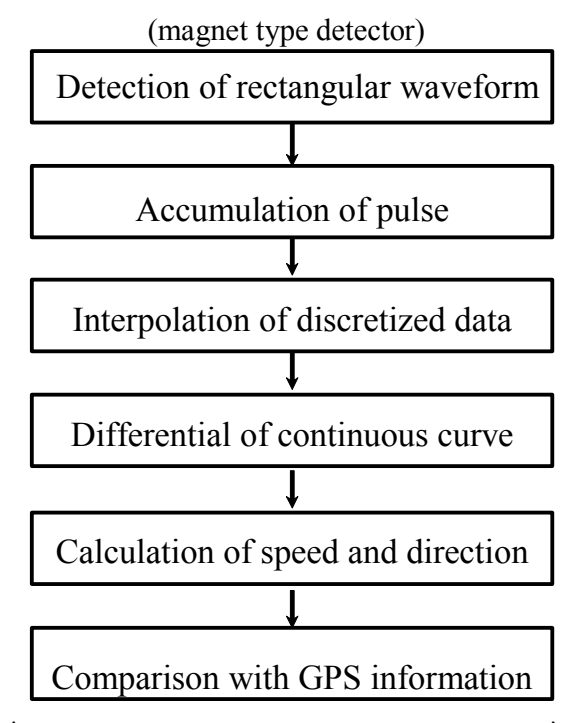

(post processing following time information)

Fig. 4 Data procedure for rotation speed evaluation

\section{GPS receiver evaluation}

Two types of D-GPS receivers were evaluated. Receiver I (Mini Max, CSI Wireless, Calgary, Alberta, Canada) uses radio beacons as differential signals. Receiver II (Crescent A100, Hemisphere GPS, Scottsdale, AZ, USA) uses satellite differential signals from a multi-functional transport satellite (MTSAT) such as the MTSAT Satellite-based Augmentation System implemented by the Japan Civil Aviation Bureau in 2007. The experiments were conducted on the roof of the main building of the Hokuriku Research Center (lat. $37^{\circ} \mathrm{N}$, long. $138^{\circ} \mathrm{E}$, Joetsu, Niigata, Japan). No obstacles to satellite communication existed at the time of measurements. The recommended minimum specific GPS data sentences, which have been standardized by the National Marine Electronics Association, were recorded as GPS information. They contain date, time, latitude, longitude, speed, direction, and other information.

The dynamic accuracy of the two D-GPS receivers was evaluated using the testbed and procedure developed for this purpose. The antenna was mounted on the arm of the platform 
at a fixed radius of $2 \mathrm{~m}$.

First, the rotation speed was gradually varied, step-wise in 11 steps. The sat frequencies were $1,2,3,6,9,12,15,20,25$, 30 , and $35 \mathrm{~Hz}$, to a circumferential speed at an antenna position of $0.05-1.80 \mathrm{~m} / \mathrm{s}$ (Fig. 3). The accuracy of velocity under constant speed gyration was analyzed at each set speed.

Second, the rotational speed was varied by changing the power frequency using an inverter. The turntable revolved at rates in the range $0.25-11 \mathrm{rpm}$, corresponding to a circumferential speed at the position of the antenna of $0.05-2.3 \mathrm{~m} / \mathrm{s}$. This range of speed is sufficient for typical farm vehicles found in a paddy field.

Data from the comparison of two receivers were recorded every second, and each receiver was tested independently. Satellite alignment was not perfectly the same in each experiment, but the effect of selecting a satellite is not discussed in this study for the following reasons. 1) Two kinds of experiments were conducted twice alternatively. Each experiment lasted for less than $10 \mathrm{~min}$. The following discussion applies to all four experiments. 2) The testbed was fixed on top of the building; nothing interrupted communication between the satellite and the GPS antenna. 3) The receivers were tested under equal conditions, considering static and dynamic accuracy. The elevation mask was fixed at $15^{\circ}$ for both receivers.

During the experiment, the pulse signals related to the rotational speed were recorded using a data logger (NR-2000; Keyence, Osaka, Japan) at a sampling rate of $100 \mathrm{~Hz}$. The signal was converted into the peripheral velocity (speed and direction) using the procedure described above. The velocity, which was calculated every second, was used as a criterion to assess the dynamic performance of the GPS receiver. Although it is technically possible to monitor the data from a GPS receiver and simultaneously detect the rotational speed in a single system, the data from the GPS receiver could experience some time delay due to calculations inside the receiver and external communications. Therefore, the data were integrated after each time stamp by post-processing to compare them more accurately.

The static accuracy was also tested for comparison with the dynamic accuracy. The location of each receiver's antenna was fixed, and the data from each receiver were recorded every $5 \mathrm{~s}$ for $24 \mathrm{~h}$.

\section{Results and Discussion}

\section{Static accuracy}

Figure 5 shows the results of GPS accuracy under static conditions for comparison with the following dynamic accuracy data. For Receiver I, 95\% of the measurements fell within a circle with a radius of $2.34 \mathrm{~m}$. For Receiver II, this radius was $0.64 \mathrm{~m}$.

\section{Dynamic accuracy}

Figure 6 shows an example of data acquired under step-wise change of speed. The GPS data were more unstable. The deviation from the criterion, which was obtained by monitoring turntable movement on the testbed, can be treated as a speed error.

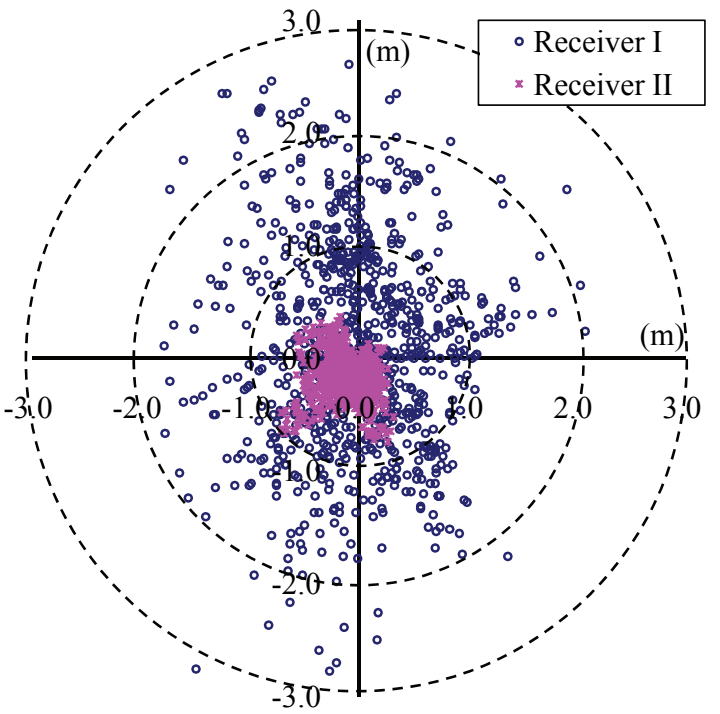

Fig. 5 Distribution of measurement points under static conditions for each receiver

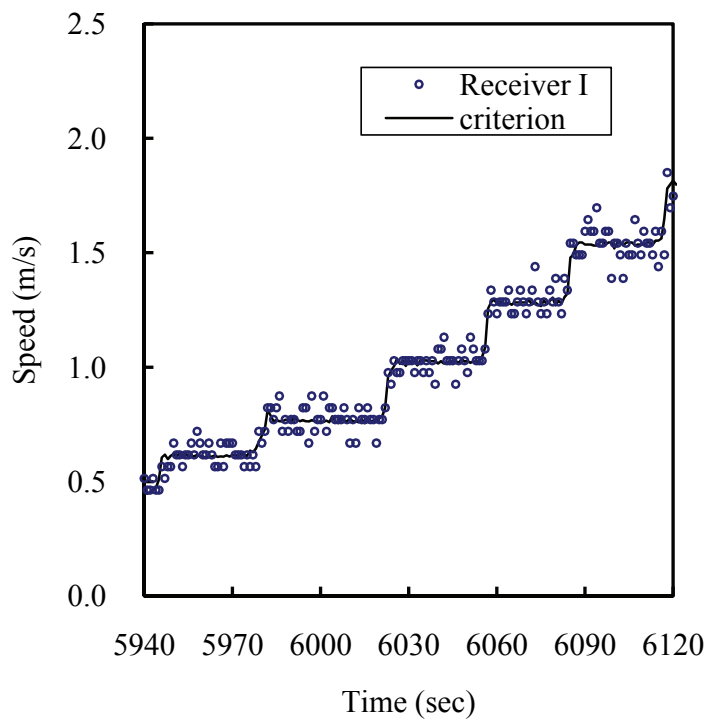

Fig. 6 Step-wise increase in speed (criteria and GPS information)

The root mean square error (RMSE) of speed increased with the increase in speed. However, a clear relationship was not observed (Fig. 7). The relative error was smaller than 
$10 \%$ when the speed was higher than $0.45 \mathrm{~m} / \mathrm{s}$ in both GPS receiver. The averages speed RMSE were 0.037 and 0.044 $\mathrm{m} / \mathrm{s}$ for Receiver I and II respectively. The regression line between speed and the speed RMSE with Receiver II was located above that of Receive I. We considered that the speed from Receiver I was more accurate than that from Receiver II.

The RMSE of the sine of direction was less than 0.175 , which corresponds to an angle of about $10^{\circ}$, except when the speed was slower than $0.15 \mathrm{~m} / \mathrm{s}$ (Fig. 8). The averages RMSEs of the sine of direction were 0.124 and 0.182 for Receiver I and II, respectively. We considered that the direction from Receiver I was more accurate than that from Receiver II.

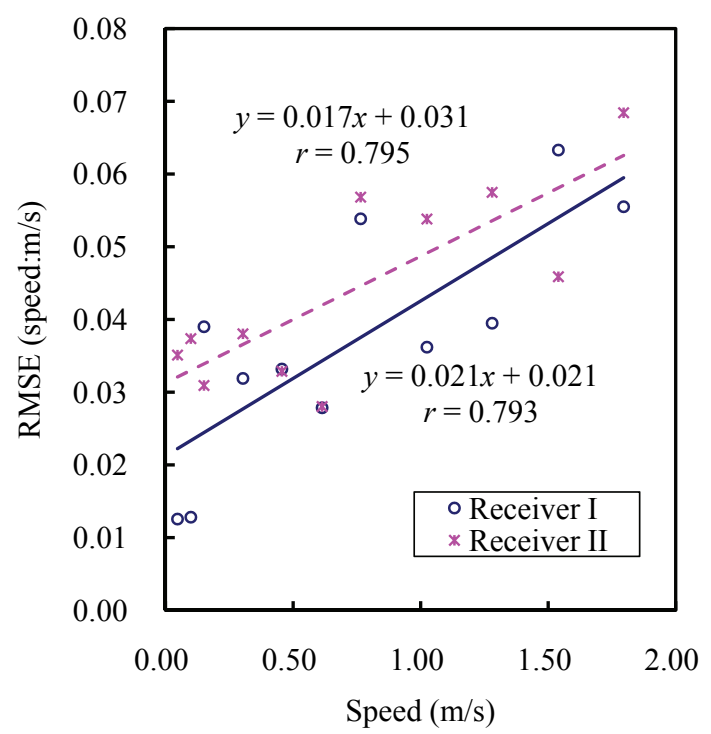

Fig. 7 Root mean square error of speed for each speed

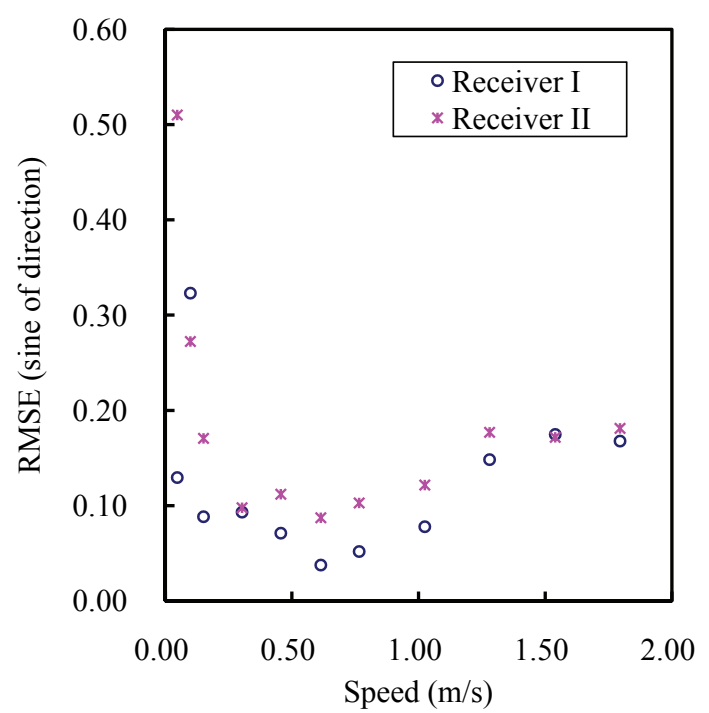

Fig. 8 Root mean square error of the sine of direction for each speed
Figure 9 shows the locations measured by the two GPS receivers during dynamic error testing. These plots do not form complete circles even though the antennas followed a curved path on the test platform. The deviation from the perfect circular shape was caused by GPS error. Figure 10 shows the speed and direction of the GPS Receiver I during dynamic error testing, including the random movement introduced by the test platform. The solid lines in the graph represent the actual expected values calculated for the antenna using the method described above.

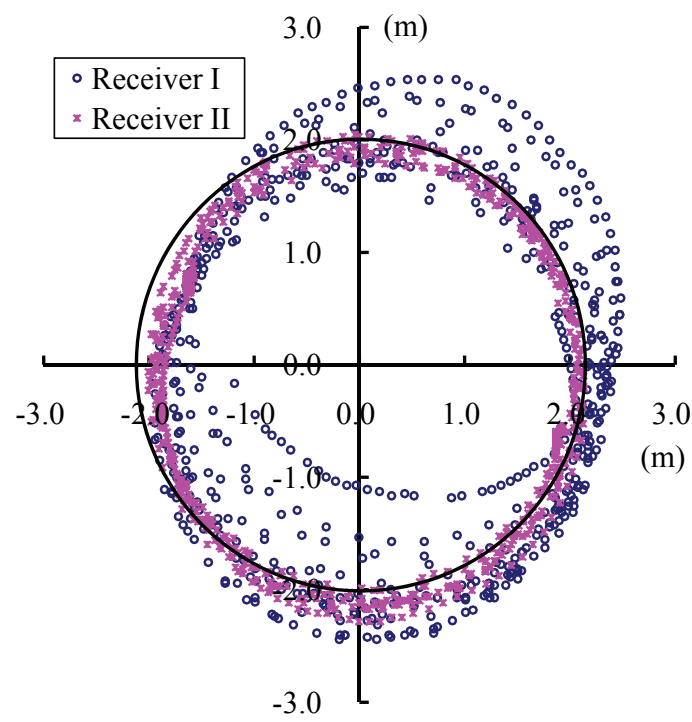

Fig. 9 Measurement points in the dynamic condition for each receiver (black line is the expected path.)

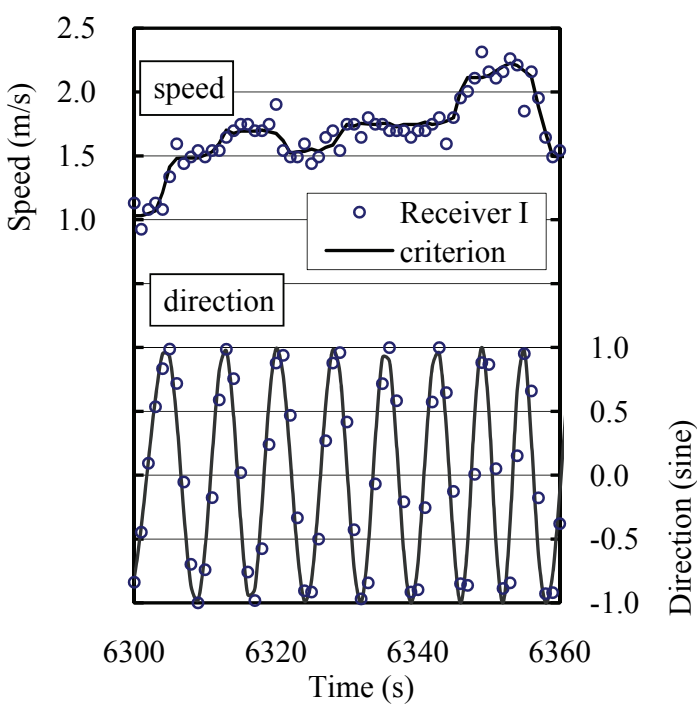

Fig. 10 An example of acquired data (speed and direction) 
Dynamic position accuracy was not evaluated using the deviation from the ideal circular path. The measured value from the testbed was used. The deviations for Receiver I were more concentrated around the true location than those for Receiver II. Even so, some deviations for Receiver I were larger than $1 \mathrm{~m}$ (Fig. 11).

As shown in Figure 11, the radii of the circles that included $95 \%$ of the measurements by Receivers I and II were 1.39 and $1.71 \mathrm{~m}$, respectively. Dynamic error was smaller with Receiver I than with Receiver II.

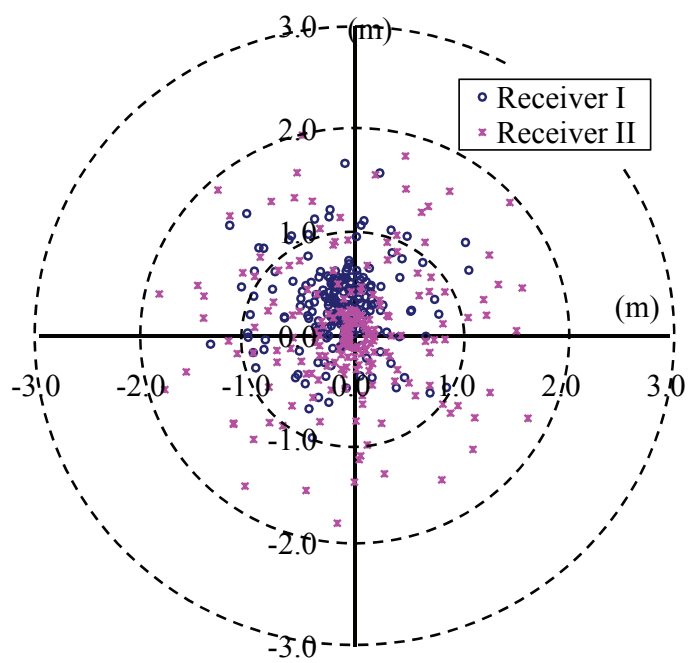

Fig. 11 Distribution of deviation from each criterion point under the dynamic condition

The deviation from the expected path of Receiver II was smaller than that of Receiver I (Fig. 9). The dynamic error in Receiver II should be lower than that of Receiver I, but Figure 11 does not show this. This contradiction indicates that the calculated error was not affected by cross-track error, but by track error. The acquired position from Receiver II tended to be delay against the measured position from the testbed. Excess filtering may be one reason why this was observed, although the data processing inside the receiver is unclear. Filtering may prevent a proper evaluation of accuracy depending on the condition, but such an influence regarding a track error could not be discussed without using the developed testbed and data processing.

The RMSE of the speed measured by Receivers I and II were almost the same, 0.067 and $0.068 \mathrm{~m} / \mathrm{s}$, respectively. The error distributions for the speed were also similar. More than $95 \%$ of the speed differences were less than $0.1 \mathrm{~m} / \mathrm{s}$, as shown in Figure 12. The random movement errors were larger than those with constant speed gyration. It was assumed that the GPS receiver response affected dynamic accuracy. The RMSE of speed was not related to actual speed (Fig. 13). The result was the same under constant speed gyration.

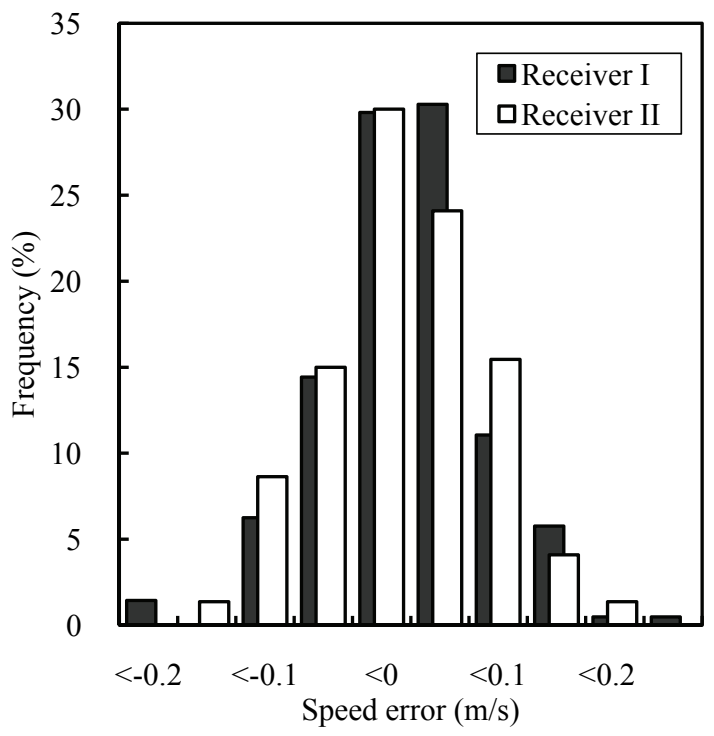

Fig. 12 Distribution of speed errors

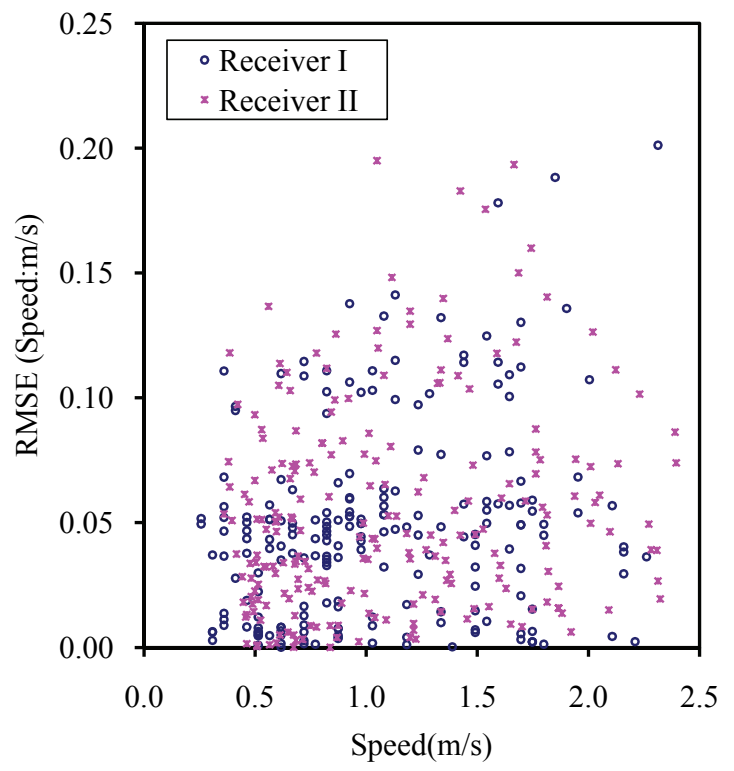

Fig. 13 Root mean square error of speed for each speed under random movement

Figure 14 shows the error distribution for the sine of the direction. The RMSE of direction (sine of the angle) of Receiver I was 0.168 , corresponding to an angle of $9.7^{\circ}$. This was 0.29 for Receiver II, corresponding to an angle of $16.9^{\circ}$. More than $95 \%$ of the differences of the sine of the angle from Receiver I were less than 0.3 , which corresponds to an angle of $17.5^{\circ}$. The error distribution of the sine of the angle from Receiver II was different. More than $15 \%$ of the differences were larger than 0.4 , which corresponds to an angle of $23.6^{\circ}$ 
The dynamic accuracy trend of the GPS receivers was completely different from the static accuracy trend. The static accuracy result shows that Receiver II had better accuracy than that of Receiver I, but the dynamic accuracy experiment did not show the same result. The speed and direction accuracies under dynamic conditions were the opposite of those under static conditions.

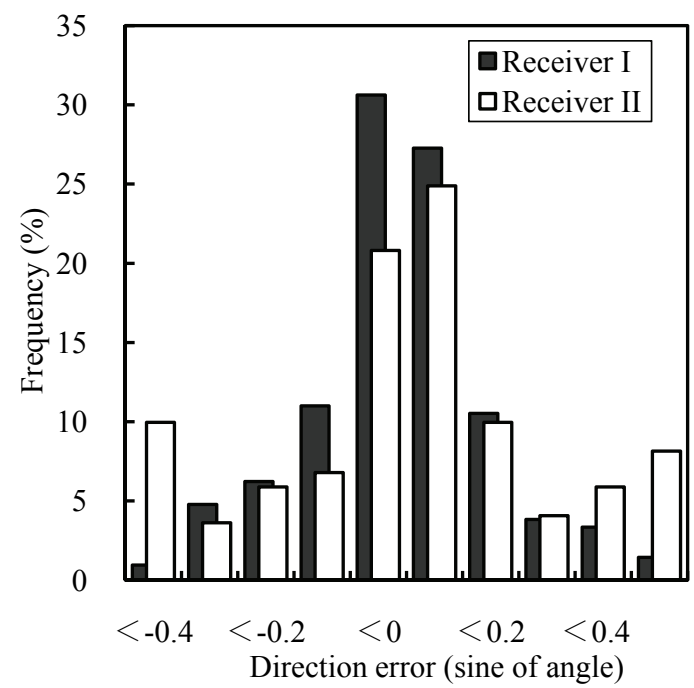

Fig. 14 Error distribution for the sine of the direction

This study did not determine the cause for this contradiction, because position calculation and signal processing are not the same in each receiver. For example, we inferred that signal filtering or post processing may be influencing the accuracy in each condition. The details of these procedures are not usually open to the public. The main point is that our attempt showed difference between static and dynamic accuracy.

\section{Further study and development}

One of the main aims of this research was to evaluate the difference between the static and dynamic accuracy of GPS receivers. Problems still exist in determining the dynamic accuracy that should be addressed in future research.

We tested two different types of D-GPS receivers. One receives radio beacon signals, and the other receives satellite signals for differential corrections. This study showed that the static and dynamic accuracies of GPS receivers and their trends are not the same. Thus, the study indicates the importance of evaluating static and dynamic errors in GPS receivers for accurate results. This may not apply to all GPS receivers. GPS accuracy is affected by various satellites factors and atmospheric conditions. The present study did not focus on the internal operation of the receivers (e.g., filtering of the signal or post-processing of the calculated result) although the receivers were tested under the same conditions during the static and dynamic experiments.

Two methods can be used to evaluate the dynamic accuracy of GPS receivers: on a vehicle or on a testbed. Vehicle testing involves placing multiple receivers on a test vehicle that is driven over a predetermined course. Testbed testing is conducted using a fixed testing setup. This work involves simple testing of static and dynamic position errors using the testbed system. Stombaugh et al. (2002) outlined the advantages and disadvantages of both types of testing. The receiver path is known accurately and can be replicated using a testbed, although this could make simulation of actual field operations and maneuvers difficult. Vehicle testing simulates typical field operations and maneuvers more closely, allowing comparisons of receiver performance for actual field conditions. However, the results can be affected by the skill of the vehicle operator, and the same path cannot be retraced exactly in multiple experiments.

The testbed and data processing procedure described here are unique and can be used to monitor small motions as a criterion for evaluating the dynamic accuracy of GPS receivers. The test method and conditions must be considered when determining the dynamic accuracy of GPS receivers. Chosa et al. (2007b) conducted both vehicle testing and testbed evaluation of GPS receiver accuracy. Several types of test may be required to determine receiver accuracy, and these methods need to be standardized. We are confident that our research has contributed to standardizing the method of evaluating the dynamic accuracy of GPS receivers.

\section{Conclusions}

An experimental testbed and data analysis procedure for evaluating the dynamic performance of GPS receivers was developed. Two types of D-GPS receivers were tested and their dynamic accuracy was determined.

1) The testbed consisted of a turntable driven by a three-phase squirrel-cage induction motor. The GPS antenna was fixed to the turntable to evaluation the dynamic accuracy of the receivers. The angular position was detected by a magnet-type detector, and the discretized detector output was interpolated by post-processing to enable monitoring of small turntable movements. The calculated movement of the turntable was used as the standard criterion for evaluating the movement of the GPS antenna.

2) The position errors under static conditions were 2.34 and $0.64 \mathrm{~m}$ for the two receivers, and were 1.39 and $1.71 \mathrm{~m}$, respectively, under dynamic conditions. The dynamic accuracy was different from the static accuracy.

3) The speed errors were 0.067 and $0.068 \mathrm{~m} / \mathrm{s}$, and the direction errors were $9.7^{\circ}$ and $16.9^{\circ}$ for the two receivers, respectively. 
4) These errors were evaluated using the testbed and data analysis procedure that we developed for the purpose. The dynamic accuracy trend of the GPS receivers was completely different from the static accuracy trend. Methods of evaluating GPS receiver dynamic accuracy should be standardized for practical applications in agriculture.

\section{References}

Chosa, T., Omine, M., Miyama, D., Morimoto, E. and Shibata, Y. 2007a. A granule applicator control system using global positioning system velocity sensor. Journal of JSAM 69(6): 84-90.

Chosa, T., Omine, M., and Itani, K. 2007b. Dynamic performance of global positioning system velocity sensor for extremely accurate positioning. Biosystems Engineering 97: 3-9.

Ehsani, M. Reza, Sullivan, M., Zimmerman, T., and Stombaugh, T. 2003. Evaluating the dynamic accuracy of low-cost GPS receivers. ASAE Meeting Paper No. 031014. ASAE, St. Joseph, MI, USA.

Han, S., Zhang, Q., Noh, H. and Shin, B. 2004. A dynamic performance evaluation method for DGPS receivers under linear parallel-tracking applications. Transactions of the ASAE 47(1): 321- 329.

Institute of Navigation (ION). 1997. Institute of Navigation Standards: ION STD 101: Recommended test procedures for GPS receivers. ION, Alexandria, VA, USA.

Nagasaka, Y., Naonobu Umeda, N., Kanetai, Y., Taniwaki, K. and Sasaki, Y. 2004. Autonomous guidance for rice transplanting using global positioning and gyroscopes. Computers and Electronics in Agriculture 43(3): 223-234.

Stombaugh, T., Shearer, S., Fulton, J., and Ehsani, M. 2002. Elements of a Dynamic GPS Test Standard. Paper No. 021150. ASAE, St. Joseph, MI, USA.

Stombaugh, T., Cole, J., Shearer, S. and Koostra, B. 2005. A test facility for evaluating dynamic GPS accuracy. In: Precision Agriculture '05, eds. J. V. Stafford, 605-612. Waganingen: Wageningen Academic Publishers.

Taylor, R.K., Schrock, M.D., Bloomfield, J., Bora, G., Brockmeier, G., Burton, W., Carlson, B., Gattis, J., Groening, R., Kopriva, J., Oleen, N., Ney, J., Simmelink, C. and Vondracek, J. 2004. Dynamic testing of GPS receivers. Transactions of the ASAE 47(4): 1017-1025.

(Received: 22. December.2009, Accepted: 22. March.2011) 